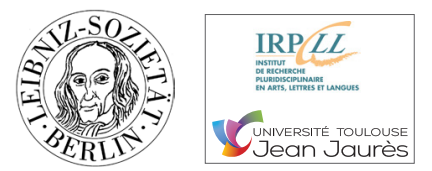

\title{
Reflexionen anlässlich der Verabschiedung der ersten Generation Studierender des Masters Kulturwissenschaften I Cultural Studies an der Université Paris Nanterre (2016-2018) ${ }^{1}$
}

\author{
Vom Eingeholtwerden durch die politische Realität oder Nanterre au joli mois de mai
}

\section{Katja Schubert}

Université Paris Nanterre

Fangen wir im Hier und Jetzt an: An der großen Mauer des Universitätsgebäudes, das von der RER-Station Nanterre Université aus zu sehen ist, hängt noch das Programm zu Veranstaltungen anlässlich des 50 . Jahrestages der Studentenrevolution von 1968. Während ich diesen Satz Anfang Juni 2018 zu einem Text schreibe, der sich als Fortsetzung des Artikels der Hauptinitiatorin des Masters Kulturwissenschaften / Cultural Studies in Nanterre, Marion Picker, Eine kulturwissenschaftliche Versuchsanordnung. Kulturwissenschaften in Frankreich (Picker 2018), versteht und eine Art Erfahrungsbericht anlässlich der Abschlussphase der ersten Generation dieses Masters werden soll, ist unsere Hochschule seit fast zwei Monaten blockiert. Ausgelöst wurde die Protestaktion durch die von der „Regierung Macron“ im Rahmen neuer Hochschulreformen vorgenommene Einführung eines neuen landesweiten Zulassungssystems für Abiturienten, des sogenannten Parcoursup (was sich vorher APB Application PostBac nannte). Parcoursup basiert einerseits auf der Grundlage der von durch die Universitäten national festgelegten attendus, also Erwartungskriterien im Hinblick auf das Niveau und die ,Kapazitäten der Abiturienten. Andererseits wurden die Klassenlehrer_innen der Abiturklassen und die Direktor_innen der Gymnasien aufgefordert, ein Dossier für jeden zukünftigen Abiturienten anzufertigen, in dem sich neben Zeugnissen auch eine fiche avenir (Einschätzung der Lehrer in Bezug auf die Studierfähigkeit des Abiturienten) sowie ein vom Abiturienten selbst formuliertes Studien-/Berufsprojekt und ein Lebenslauf befinden. Aus dieser Informationsdichte heraus soll der Abiturient seine Mehrfachwünsche für verschiedene

1 Für Marion Picker in Dankbarkeit und Freundschaft.
Studienfächer formulieren. Wie jede Hochschule hat nun Nanterre Tausende solcher komplexer Dossiers bekommen und soll, so die Direktive des Ministeriums, in minutiöser individueller Dossierprüfung, nach einem für jedes Fach entwickelten Parameter, eine Selektion und Platzierung der ,geeignetsten“ Kandidaturen für die vorgesehene Zahl von Studienplätzen in jeder Disziplin vornehmen. Dieses Verfahren soll offiziell dem weit verbreiteten Phänomen des Studienabbruchs bereits im ersten Jahr einen Riegel vorschieben und die Erfolgschancen für zukünftige Studierende erhöhen.

Seit Monaten gibt es Proteste gegen dieses System. Viele engagierte Akteur_innen der Schul- und Hochschullandschaft verweisen auf die Ungleichheit im Schulsystem und infolgedessen auf unangemessene Selektionsraster, die sich im Zulassungssystem widerspiegelten: Gymnasien beispielsweise aus gutbürgerlichen Pariser Stadtteilen bieten andere Lernvoraussetzungen - und damit oft andere Resultate im Abitur - als solche, die sich täglich mit der sozialen Heterogenität der Schüler_innen in den Klassen auseinanderzusetzen haben. Darüber hinaus könne man mit 17 Jahren, so viele Kritiker_innen, kein glaubwürdiges Studien- und Arbeitsprojekt formulieren, da man darauf während der Schulzeit nicht vorbereitet werde und auch mehr Zeit brauche, sich mit dieser Frage auseinanderzusetzen. Zugleich existierten alternative Ausbildungswege nach dem Abitur nur in geringem Maße (BTS, Brevet de technicien supérieur, zum Beispiel) oder seien nicht bekannt und/oder auch nicht, angesehen'. Zum anderen richtet sich der Protest genauso stark gegen die technische Machbarkeit im Bewerbungsverfahren selbst, das heißt die hinreichende Bearbeitung der Bewerbungsunterlagen, ohne dass ausreichend Personal dafür an den Universitäten zur Verfügung steht, da sich der Abbau von 
Arbeitsplätzen und die unzureichende Finanzierung der Hochschulen trotz wachsender Studentenzahlen in den letzten Jahren eher beschleunigt haben. Es geht also in gewisser Hinsicht um die nach Frage der Zukunft und den Aufgaben der Universitäten, aber auch um die Vision der Gesellschaft, die jungen Menschen eine Ausbildungs- und Lebensperspektive bieten muss. Die sich für diese Fragen engagierenden Studierenden und Dozent_innen begreifen darüber hinaus das geplante Auswahlverfahren als nur ein Element in einer umfassenden, von der derzeitigen Regierung angestrebten ,neoliberalen' Reformlandschaft, die Leistung, Verdienst und Elitenbildung in den Mittelpunkt stellt, während andere Kriterien zu sozialen Reformen kaum auf der Agenda vorkommen. So kam es zu dem so vielleicht nur in Frankreich anzutreffenden Phänomen, dass unter dem Stichwort der solidarischen convergence de luttes auch Eisenbahner_innen, Postbeamt_innen und Grundschullehrer_innen regelmäßig an den in Nanterre stattfindenden Vollversammlungen teilnehmen.

Blockade heißt nun, dass die Gebäude nur für Personal zugänglich sind, nicht aber für die Studierenden, dass die im Terminkalender vorgesehenen Endjahresexamina nicht durchgeführt werden konnten und dass derzeit von einem größeren Anteil des Lehrkörpers nach Alternativen der Notengebung gesucht wird, da man der Meinung ist, die Studierenden hätten ein ,Anrecht' auf ihre Noten. Die Gegner dieser Maßnahmen wiederum glauben, dass die Verweigerung von Noten das wirksamste Mittel sei, um Druck auf die universitäre und auch nationale politische Führung auszuüben, damit Parcoursup zurückgenommen und grundlegend neu über die Hochschullandschaft und deren Aufgaben in der Zukunft nachgedacht und entschieden werden könne. Im Augenblick der Niederschrift dieses Textes sind die Fronten verhärtet und ein Dialog findet nicht statt, zumal eine politische Fehlentscheidung des Präsidenten der Universität Nanterre, eine Versammlung Mitte April mit Polizeigewalt aufzulösen, diese Frontstellung auf extreme Weise zugespitzt hat. ${ }^{2}$

Die ausführliche Schilderung dieser Sachverhalte ist insofern von Bedeutung, als ein Teil der Gruppe des Masters $2^{3}$ sehr intensiv die Entwicklungen auf dem Campus und an den Universitäten landesweit mitverfolgt und sich daran beteiligt hat. Darauf komme ich gleich noch einmal zurück.

Erinnert sei hier noch einmal daran, wie es Marion Picker in ihrem Artikel bereits ausgeführt hat, dass die Einrichtung des Masters Kulturwissenschaften / Cultural Studies im Jahre 2016 in Nanterre ein Experiment darstellt im Hinblick auf

2 Nachtrag November 2018: Zu Beginn des Studienjahres im September 2018 hatten ungefähr 50.000 Abiturient_innen noch keinen Studienplatz durch Parcoursup erhalten. Bis Mitte Oktober, nachdem die Kurse schon seit mehreren Wochen angefangen hatten, tauchten in Nanterre immer wieder Nachzügler_innen auf, die in den meisten Fällen Gertauchten in Nanterre immer wieder Nachzügler_innen auf, die in den meisten Fällen Ger-
manistik nicht als Wunsch auf ihrer Liste hatten. Man hatte sie in Nanterre mit der Aussicht auf eine eventuelle Reorientierung im zweiten Semester in ein gewünschtes Fach aufgenommen, ohne zu wissen, welche realen Chancen für den Wechsel bestehen. Sie wurden in die Germanistik verwiesen, da es dort aufgrund des Rückgangs der Einschreibungen in diesem Fach relativ kleine Gruppen gibt. Dass der ein oder andere von diesen Studierenden keinerlei Deutschkenntnisse hatte, interessierte die Administration nicht. Die De batte über die Reformierung von Details von Parcoursup ist im Gange, eine fundamental Infragestellung steht im Moment nicht auf der Tagesordnung der Regierung. Eine bestimmte Zahl von Abiturient_innen ist an private Hochschulen abgewandert. Die Zahl der Studienabbrecher im ersten Semester, dem das neue Auswahlsystem entgegen steuern sollte, schein sich nach ersten Beobachtungen nicht zu verringern. Für die nächsten Jahre werden weiterhin wachsende Abiturientenzahlen erwartet.

3 Dies entspricht dem zweiten Studienjahr des Masters. die Frage, ob man diesen in den anglophonen und deutschsprachigen Ländern schon seit Jahren etablierten und doch immer wieder kontrovers diskutierten Forschungsansatz mit seinen ganz spezifischen Denktraditionen, Wissenskulturen und Debatten um die Neuorganisation althergebrachter Fächerkonfigurationen nach Frankreich importieren kann. Wolfgang Riedel hat darauf verwiesen, wie man heute den konfliktreichen Traditionen der Kulturwissenschaft Rechnung tragen kann. Die Thematisierung der Geschichte der Kulturwissenschaften sowie der wichtigen Momente des Wissenstransfers, einschließlich einer Betrachtung der sich wandelnden Kulturbegriffe, die bis zur Sattelzeit nach 1750 zurückverfolgt werden können, sind für Riedel dabei zentral (vgl. Riedel in Picker: 2018, 231).

Ein spezifisches Seminar zu diesen Entwicklungen in der Theoriegeschichte, in dem sich Studierende der Kulturwissenschaft und der Anglistik treffen, soll als eine Art Kompass und Werkzeugkasten dienen für all die anderen Kurse in interkultureller Linguistik und Literatur, Religionswissenschaft, Medienkunde, Übersetzung, Bildender Kunst sowie für die sozialwissenschaftlichen Seminare zur Anthropologie, Soziologie und Kunstgeschichte. Es handelt sich um einen ,Grundlagenkurs', dessen theoretische Reflexion im Idealfall in den anderen Fächern Anwendung findet, was von den Studierenden selbst auch nachvollziehbar sein sollte. Insgesamt besticht das Angebot des neuen Masters durch seine disziplinäre Vielfalt und ist zugleich eine intellektuelle Herausforderung für die Studierenden, die in viele Richtungen zugleich zu denken angehalten sind und im ständigen Wechsel zwischen deutscher und französischer Sprache lesen, schreiben und sprechen.

Angesichts der derzeitigen Proteste gegen die geplanten Hochschulreformen, und damit schlage ich noch einmal den Bogen zu meinen Eingangsüberlegungen, scheint die Einrichtung eines solchen Masters fast anachronistisch: Es geht hier nicht um einen Master, der die direkte Einsetzbarkeit der Studierenden auf dem Arbeitsmarkt vorgeben kann, oder der sich dafür eignet, in lückenlose Biographien und Karriereplanungen eingebaut zu werden. Denn lückenlos, ohne Pause, soll es nach Meinung der Regierung ja auch für die Abiturient_innen immer weiter gehen, die mitten in der Endphase des Abiturs dieser Tage erfahren haben, ob sie nach der ersten Bewerbungsphase im Parcoursup zu den fast 50 Prozent gehören, deren Fachwunsch erfüllt wurde oder zur anderen Hälfte, die ihr Fach nicht bekommen hat und im besten Fall auf einer Warteliste gelandet ist.

Die von uns konzipierten Kulturwissenschaften wären da hingegen viel mehr ein Raum, wo noch einmal oder zum ersten Mal eine Form der Selbstreflexion im Hinblick auf das eigene Denken, das eigene Fach und die vielschichtigen Beziehungen zwischen Wissen, Kultur, Macht und Gesellschaft stattfinden kann. Dafür braucht es Zeit.

Im Rahmen der Vorbereitung einer von den Studierenden des Masters 2 mit organisierten Journée d'études Mitte April, gedacht als eine Art abschließender ,Höhepunkt' der zwei Jahre, wo die Abschlussarbeiten vor einem Publikum von Dozent_innen und Studierenden anderer Jahrgänge 
vorgestellt werden sollten, kam es angesichts der Situation auf dem Campus zu einem Streitgespräch innerhalb der Gruppe. Die Hälfte der Masterstudierenden, die sich für die politischen Vorgänge aktiv interessierte, forderte, dass man die Journée d'études in ein offenes Atelier umwandeln solle, in dem Raum sei für Fragen, die mit der Aktualität in Zusammenhang stünden, während der andere Teil der Gruppe eindeutig sein Desinteresse an dem derzeitigen Geschehen bekundete und vor allem an der Präsentation der eigenen Arbeit interessiert war. Das Ergebnis war eine Journée d'études en deux temps: im ersten Teil wie vorgesehen die Diskussion über die Masterarbeiten und in der zweiten Hälfte der Versuch, das eigene Arbeiten und die Existenz als Studierende hier und heute in ein Verhältnis zu setzen mit den aktuellen politischen Fragen und Geschehnissen, die in Nanterre und darüber hinaus stattfinden. Die Veranstaltung fand in einem besetzten Gebäude in einem Raum statt, in dem am Morgen noch Studierende schliefen und am Abend Sprachkurse für Migrant_innen stattfanden, die seit zwei Jahren in Nanterre durchgeführt werden. Ohne dass dies intendiert war, zeigte dieser auch räumlich ,ungewöhnliche“ Kontext auf seine Weise eine feine Spur auf von der Möglichkeit der Herstellung von neuen Konfigurationen im Lern- und Lehrbereich, wo plötzlich, auf fast überraschende Weise, Elemente zusammenkamen, in denen Lehre, Forschung, Politik und Engagement einander berührten und auch in der Perspektive der Zusammengehörigkeit diskutiert wurden. Diese Besonderheit und Vielfalt zogen sich in gewisser Hinsicht als Leitmotiv durch die ganze Journée d'études: sieben beteiligte Dozent_innen und ebenso viele Master-Studierende sowie ein kleines Publikum dazugekommener Studierender hörten einander zu und tauschten sich intensiv über sehr viele Fragen aus. Wir hatten uns die Zeit und den Raum dafür gegeben. Da fast alle Arbeiten zahlreiche Kommentare von den Kolleg_innen erhielten, konnten die Studierenden auf einmalige Weise eine wirklich reichhaltige Reflexion zu ihrer ganz persönlichen Arbeit mit nach Hause nehmen. Und auch der zweite Teil des Tages ließ nicht an Intensität nach: Das Gespräch über die gegenseitige Selbstwahrnehmung und Analyse der Situation, von Studierenden und Lehrenden, in Nanterre, in Frankreich, in der Epoche von Populismus und der Auflösung bestimmter Begriffe und Realitäten ,des Politischen', auch im Rahmen der Globalisierung, ließ uns von Dingen hören und über sie sprechen, wie wir es in einer solchen Konfiguration und Offenheit noch nicht erlebt hatten. Zugleich spiegelten sich darin zentrale Dimensionen der Disziplin Kulturwissenschaft selbst wider. Es sei deshalb noch einmal betont, dass Fragen, die derzeit im Bereich der französischen Hochschulpolitik, aber auch darüber hinaus in der Gesellschaft gestellt werden, in gewisser Hinsicht auch im Mittelpunkt des Faches Kulturwissenschaft stehen. Im Wissenschaftsbegriff der Kulturwissenschaften spielen die Kombination von Heterogenem und die Pluralität von Wissensformen, Lebensformen und kulturellen Orientierungen eine entscheidende Rolle. Das eigene Handeln als kulturell Bedingtes verbindet sich mit Forschungen zur Geschichtlichkeit der eigenen Praxis und des Zugangs zu Wissen und
Kultur. Sprache, Medien, Bilder, Symbolbildungen aller Art und Institutionen werden als unterschiedliche, konfligierende wie ausdifferenzierte, machtgestützte wie subversive Codierungen ausgelegt, die konstitutiv für die gesellschaftlichen Wirklichkeiten sind. Die Verbindung zwischen symbolischen und realen Ordnungen erfahren demnach höchste Aufmerksamkeit. Reflexion mit politischem Handeln zu verbinden, ist auch einer bestimmten ,deutschen' Tradition der Kulturwissenschaften nicht fremd.

Darüber hinaus brauchen Transgression und Übersetzung zwischen Lebenswelten und Wissensordnungen, zwischen Textlektüren und gesellschaftlichen Handlungspraktiken aber auch Mediator_innen. Wir erleben dies im Moment auf unserem Campus, und zugleich war diese Perspektive, ,Mittlerpersonen' ausbilden zu können, eine Motivation für den Aufbau dieses Studiengangs.

\section{ZWEI JAHRE VOLLER ERFAHRUNGEN UND NEUER FRAGEN}

Trotz des enormen Gewichts, das die aktuellen Ereignisse in Nanterre für den Blick auf die ,Kuwi' in den letzten Monaten angenommen haben, ist es für die eigene Verortung wichtig, noch einmal Rückschau zu halten auf bedeutende Momente und Erfahrungen mit dem neuen Studiengang, der auch weiterhin ein experimentelles Labor bleiben wird.

Festzuhalten ist, dass die Studierenden aus ihrem zweiten Semester mit Praktikum und Sommersemester in Frankfurt/ Oder, Halle und Tübingen mit sehr positiven Erfahrungen in den Master 2 nach Nanterre zurückgekehrt sind. Die rapports de stage legten ein Zeugnis von der Bedeutung der Praktika ab, so dass die Anfrage zugleich auch für den Master 2 kam, ob man in dessen Rahmen ein zweites Praktikum integrieren könnte. In der Tat haben Studierende des Masters 2 sich nach dem Ende der Kurse im April ein zweites Praktikum zwischen Mai und August 2018 gesucht, da sie bis dahin noch an der Universität eingeschrieben, also auch versichert sind. Für alle Studierenden war der Start an der deutschen Universität zunächst schwierig, da sie Zeit brauchten, das neue Universitätssystem kennenzulernen und ihren Platz darin zu finden. Die geforderten Sprachkenntnisse waren nicht immer gegeben. Gleichzeitig waren sich alle Erasmusstudierenden einig, dass die Einführungswoche an allen Universitäten sehr gelungen war und eine gute Basis für sie bildete, um für fast alle auftretenden Probleme einen Ansprechpartner zu finden und erste Kontakte zu schließen. Es scheint, dass eine solche Initiation tatsächlich die ersten größten Ängste beseitigen half, was wiederum ein wichtiger Anstoß für die Pflege unser eigenen ,Willkommenskultur' an französischen Universitäten ist, die im Vergleich zu Deutschland weniger ausgebildet ist. Nach dieser ersten steilen Hürde konnten die meisten Studierenden dann mehr und mehr ihr ,Hineinwachsen' in das deutsche System genießen: Das Kennenlernen einer anderen Universitätslandschaft und Studienkultur, andere Beziehungen zwischen den Studierenden und den Dozent_innen, 
eine Freiheit in der Meinungsäußerung im Seminar sowie die Herausforderung zu meistern, längere Hausarbeiten auf Deutsch geschrieben zu haben - das sind besonders positive Faktoren, die auch für die Weiterarbeit im Master 2 entscheidend sind. Manch einer hat auch Überraschungen erlebt, mit denen er oder sie nicht rechnete: dass man in Frankfurt/Oder zum Beispiel intensiv osteuropäische Sprachen studieren kann, wurde für eine Studentin zum Anlass, ihren Aufenthalt gleich noch um ein Semester zu verlängern.

Die Tatsache, dass wir die ,Ausreisenden' zur freien Kurswahl, entsprechend ihren Interessen, für das Sommersemester ermutigt hatten, führte in der Tat bei dem ein oder anderen Studierenden zu einer Profilierung seines oder ihres Studieninteresses, wofür dann ein Anschluss im Master 2 in Nanterre gesucht werden musste, was nicht immer ganz leicht war. Dabei war erfreulich, dass im Master 2 immer wieder Kurskommentare kamen, in denen die Studierenden versuchten, Brücken zu Studieninhalten aus ihrem Auslandssemester zu schlagen. Ein Beispiel sind die europäischen Erinnerungskulturen oder Kurse zu Gender- und Postcolonial Studies, wofür in Deutschland ein breites Angebot besteht und welches Studierende dann auch für ihre Masterarbeit fruchtbar gemacht haben.

Als positiv empfunden wurde von den Studierenden auch, dass im Master 2 in Nanterre mehr Kurse auf Deutsch gehalten werden als im Master 1: Ein Themenschwerpunkt im zweiten Jahr ist Religion und Gesellschaft, hinzu kommen Kurse zur Malerei, deutsch-französische Wissenschaftskulturen, Berlin und Literatur nach 1989. Für nächstes Jahr sind auch Kurse zur Frage der Medien und der Medialität, Schlüsselbegriffe der Kulturwissenschaften vorgesehen: Die Kolleginnen werden Lernmodule zur Jazzmusik und zum zeitgenössischen deutschsprachigen Kino, zu TV-Serien und zur Photographie vorstellen. Diese kleine Liste zeigt aber auch, dass sich der Master Kulturwissenschaften momentan in Nanterre vielleicht mehr durch ein Angebot ,neuerer', weniger konventioneller Themen charakterisiert, als durch eine kulturwissenschaftliche Theorielandschaft, auf deren Basis die jeweiligen Themenseminare angeboten werden. Selbstkritisch muss hier vermerkt werden, dass die theoretische Fundierung, die im ersten Semester durch den erwähnten Einführungskurs in die Entwicklung und Fragestellungen der Cultural Studies einerseits und der Kulturwissenschaften andererseits auf einer soliden Basis erfolgt, parallel in anderen Kursen und auch in den folgenden Semestern noch stärker ausgebaut bzw. angewendet werden sollte. Das wäre eine der Herausforderungen für die nächsten Jahre. Dazu sollte für die Studierenden auch eine stärkere Heranführung an die Sozialwissenschaften geleistet werden, indem ebenfalls nächstes Jahr als Neuheit ein Lektürekurs auf Deutsch zu sozialwissenschaftlichen Grundlagentexten (Weber, Elias, Simmel, Bourdieu, Biographieforschung, Oral history etc.) angeboten wird, auch damit die Studierenden besser gerüstet sind für ihre Masterkurse in Soziologie, Anthropologie und Kunstgeschichte.

Verbindungen des Masters zum Bereich der Forschung konnten im Master 2 durch die Teilnahme der Studierenden an zwei Tagungen im November 2017 hergestellt werden, die wiederum im Zusammenhang standen mit den angebotenen Seminaren: ein in Nanterre selbst stattfindendes, von mir und zwei Kolleginnen organisiertes Kolloquium zu Protestants et juifs entre ,affinités électives' et dialogue impossible? und die Tagung Interkulturelle Dialoge in der Kolonialzeit und der postkolonialen Periode. Literarische und kulturelle Repräsentationen - Orient, Maghreb, Westafrika von 1830 bis heute am Deutschen Historischen Institut in Paris. Für die vortragenden Kolleg_innen, die zugleich Lehrende im Master sind, stellt sich hier ganz konkret die Frage, wie man die eigene Forschungstätigkeit mit der Lehre im Master noch stärker verbinden kann. Wie wenig zugänglich einem Teil der Studierenden zum Teil auch am Ende des Studiums und noch in der Phase der Redaktion der eigenen Masterarbeit die wissenschaftliche Arbeit erscheint und wie sich dies auch in konkreten Schreibproblemen äußert, hat sich ebenfalls in den letzten Monaten gezeigt und war auch Gesprächsthema auf der bereits erwähnten Journée d'études im April 2018. In diesem Zusammenhang kam auch der Wunsch auf, ein Atelier zum wissenschaftlichen Schreiben anzubieten.

Zum aktuellen Zeitpunkt sieht es so aus, dass eine Studentin eine Doktorarbeit anvisiert und dies in einer eventuellen Partnerschaft mit der Universität Frankfurt / Oder. Die anderen Studierenden sehen sich eher im weiten Feld der métiers de la culture, worin der Master einen guten Einblick geben konnte. Die in die entsprechenden Kurse eingeladenen Akteur_innen aus diesen Bereichen konnten teilweise konkrete Hilfestellungen geben, wie beispielsweise eine Kollegin von der Universität Paris 4 Sorbonne, die viele Jahre in der diplomatie culturelle in Deutschland und Großbritannien tätig war und im Rahmen des CIERA (centre interdisciplinaire d'études et de recherches sur l'Allemagne) regelmäßig Intensivseminare zur Arbeitssuche in kulturellen Einrichtungen anbietet und die Studierenden ermutigte mit der Aussage, dass man gerade in Deutschland derzeit im Kulturbereich Arbeit finden könne. An diesen ,Professionalisierungs-Kursen“ nahmen erfreulicherweise auch Studierende der Universität Paris 8 teil, so dass zurecht von einem Studierendenaustausch zwischen Nanterre und Paris 8 gesprochen werden kann, da die Nanterroiser Studierenden für zwei Übersetzungskurse nach Paris 8 gehen. Auch diese Kooperation sollte sich in den kommenden Jahren noch weiter vertiefen. Im selben Zusammenhang hat sich auf dem Campus auch die Existenz der BDIC (Bibliothèque de documentation internationale contemporaine), die nun La contemporaine heißt, als wahre Schatzgrube entpuppt. Nicht nur bietet sie originelle Formen von Praktika an. Sie kann auch mit Originalen aufwarten wie Bilder aus der Berliner Sezession oder politischen Zeitschriften der 1920er Jahre, die den Studierenden unbürokratisch zugänglich gemacht werden. Nicht zuletzt könnte La contemporaine im Grunde jedem historisch oder politisch interessierten Studierenden eine weite Themenpalette für die Masterarbeit anbieten. Für 2018/19 ist die Erarbeitung von zwei Ausstellungen mit Hilfe der Studierenden geplant: eine erste zum deutschen Anteil des Archivs, dessen Präsentation 
auch als Online-Ausstellung konzipiert werden soll, und wahrscheinlich eine weitere Ausstellung zu 30 Jahren Wiedervereinigung, die durch Materialien und Kontakte mit der Bundeszentrale für Politische Bildung unterstützt wird.

\section{WEITERARBEITEN}

Die Versuchsanordnung ,Kuwi', wie wir sie vor zwei Jahren gestartet haben, kann also auf einen reichen Erfahrungsschatz zurückblicken und stellt zugleich viele weitere Fragen. Das Programm, und das bestätigen auch die Studierenden, ist anspruchsvoll und intellektuell herausfordernd, es öffnet unbekannte Horizonte durch neue Disziplinen, deren Entdeckung mehr ins Gewicht fällt als die Schwierigkeiten, die dabei auch auftreten. Ein point fort ist ohne Zweifel das zweite Semester mit Praktikum und Sommersemester in Deutschland. Praktikumsstellen wurden gefunden in Museen, Kulturzentren, wissenschaftlichen Einrichtungen, im Archiv und in einer Zeitungsredaktion. Die Kolleg_innen, die ,Kuwi‘ unterrichten, zeigten sich in den beiden Jahren aufgeschlossen und interessiert, einerseits an der neuen Herausforderung, kulturwissenschaftlich zu arbeiten, andererseits auch immer wieder an Gesprächen zur Konzeption und zu möglichen Verbesserungen und Veränderungen des Parcours und am Werdegang der Studierenden in den zwei Jahren. Mehrere Kurse funktionierten teilweise nach dem Prinzip der deutschen Ringvorlesung, was eine neue Dynamik auch unter Kolleg_innen schaffen kann. Ansätze neuer Vernetzungen innerhalb von Nanterre, aber auch mit Kolleg_innen anderer Universitäten oder mit Akteur_innen/Institutionen der métiers de la culture wurden geschaffen und können ausgebaut werden. Dazu zählt auch die Nutzung des Erasmus-Plus-Programms, um langfristig zwischen den Partneruniversitäten auch einen Dozent_innen-Austausch zu organisieren, der für die Beteiligten ebenfalls eine Quelle der Inspiration sein könnte und zur Vertiefung der Beziehungen beitragen würde. Eine Anfrage aus Salzburg für eine ,Kuwi'-Partnerschaft soll noch vor den Sommerferien positiv beschieden werden.

Die Professionalisierung im Master weiter stark zu machen, entspricht den geäußerten Bedürfnissen von Seiten der
Studierenden, da die Sorge, nach dem Master ohne Arbeit dazustehen, immer wieder präsent ist. Auch Studienreisen mit einer ,beruflichen Komponente', wie beispielsweise ins Literaturarchiv nach Marbach, wo Einführungen in die Archivarbeit für Masterstudierende angeboten werden, sind denkbar. Werbematerial für unseren Master-Studiengang wurde dieses Jahr auch an verschiedene Romanistikabteilungen in Deutschland verschickt, in denen wir darauf aufmerksam machen, dass man in Nanterre Kulturwissenschaften in der Kombination von deutscher und französischer Sprache studieren kann, was vielleicht langfristig, falls der Master eine kleine Bekanntheit auch in Deutschland bekommen könnte, attraktiv ist. Die Werbung an französischen Universitäten ist nicht einfach, da sie leicht als „Konkurrenzveranstaltung“ empfunden wird und die Kolleg_innen selbst sich ebenfalls um genug Einschreibungen für die eigenen Masterkurse kümmern müssen.

Was bleiben wird von den Bildern des Protestes in diesem Frühling 2018 in Nanterre ist aber auch immer wieder dieses: eine Sehnsucht, anders zusammen zu lernen, zu forschen, die Universität als Lebensort gestalten zu können, sich gemeinsam zu engagieren, die Hochschule als Teil der Gesellschaft stärker sicht- und fühlbar zu machen, der intellektuellen Neugier nachgehen zu können, Zeit zu haben, das eigene Denken zu entwickeln, sich als Studierender nicht als Manövrierteilchen einer zum Unternehmen gewordenen Einrichtung zu fühlen, in der nur von réussite gesprochen wird, ohne dass das Wort anders als durch Leistungspunkte definiert wird. In seinen besten Momenten sind wir mit dem Master Kulturwissenschaften mittendrin in diesem Aufruhr, in dieser Vielfalt.

Nachtrag November 2018: Der Master Kulturwissenschaften hat in diesem Studienjahr 2018/19 eine erfreuliche Verstärkung durch Studierende des neuen "Master International“ in Nanterre erhalten, in welchem zwei Sprachen als Hauptfächer studiert werden. Der Anteil der Germanistik wird dabei vom Master Kulturwissenschaften abgedeckt. Mehrere neue Studierende sind nach ihrem Auslandssemester mit sehr guten Deutschkenntnissen in unseren Master gekommen. Die Partnerschaft mit Salzburg wurde abgeschlossen. Zwei aktuelle Themen stehen dieses Jahr besonders im Mittelpunkt: der Klimawandel in kulturwissenschaftlicher Perspektive und 30 Jahre Wiedervereinigung.

\section{Bibliographie}

Picker, Marion (2018): „Eine kulturwissenschaftliche Versuchsanordnung. Kulturwissenschaften in Frankreich“, in Ettrich, Jenny / Mäder, Marie-Therese (Hg.), Dialogpotenziale kulturwissenschaftlicher
Forschung in den Fremdsprachenphilologien, Berlin, Peter Lang, 227-237. 